\title{
Reduced Reactivity of Aged Au Nanoparticles in Ligand Exchange Reactions
}

\section{Victor Chechik}

\section{Supporting Information.}

\section{Synthesis and characterisation of Au nanoparticles}

A $1 \% \mathrm{w} / \mathrm{w}$ aqueous solution of hydrogen tetrachloroaurate $(3.57 \mathrm{~mL}, 0.1 \mathrm{~mol})$ was mixed with a solution of tetraoctylammonium bromide $(280 \mathrm{mg})$ in toluene $(10 \mathrm{~mL})$ and stirred vigorously until all $\mathrm{Au}$ (III) was transferred to the organic phase (e.g., the aqueous phase became colorless). A $10 \% \mathrm{w} / \mathrm{w}$ solution of n-butanethiol in toluene (90 $\mathrm{mg}, 0.1 \mathrm{~mol}$ ) was added to the reaction mixture. A solution of sodium borohydride (40 $\mathrm{mg})$ in water $(1 \mathrm{~mL})$ was then added to the reaction mixture with vigorous stirring. Reduction of $\mathrm{Au}(\mathrm{III})$ to $\mathrm{Au}(0)$ and formation of nanoparticles occurred instantaneously, as visualized by color change from yellow to dark brown. At this stage, most literature recipes leave the reaction mixture stirring for extended periods of time (several hours). In a control experiment, we found that prolonged stirring reduces the reactivity of nanoparticles towards exchange. To minimize the ageing of the nanoparticles during the synthesis step, the reaction time was therefore reduced to 5 min only. After this period, the mixture was evaporated using rotary evaporator, dissolved in toluene $(3 \mathrm{~mL})$ and precipitated with methanol $(45 \mathrm{~mL})$. These operations were performed rapidly to reduce ageing. The precipitate was collected by centrifugation, washed with methanol ( $3 \times 50 \mathrm{~mL}$, washing was aided by sonication) and dried.

In calculating the concentration of nanoparticles for the exchange reaction, the composition of the particle was assumed to be $\mathrm{Au}_{500}\left(\mathrm{C}_{4} \mathrm{H}_{9} \mathrm{~S}\right)_{150}$. The number of $\mathrm{Au}$ atoms in the nanoparticle core was calculated by the formula: $N_{\mathrm{Au}}=\frac{\pi}{6} D^{3} \bar{n}_{\mathrm{Au}}^{\mathrm{fcc}}$, where $N_{\mathrm{Au}}$ is the number of $\mathrm{Au}$ atoms in the particle, $D$ is the particle diameter measured from TEM images, and $\bar{n}_{\mathrm{Au}}^{\mathrm{fcc}}=59.2$ (packing density of $\mathrm{Au}$ ). ${ }^{1}$ The number of ligands was calculated by comparison with the literature value ${ }^{2}$ an elemental analysis of one representative batch of butanethiol-protected nanoparticles was consistent with this calculation.

UV spectra were run in chlorobenzene solution $(1 \mu \mathrm{M})$. The spectra were fitted to an empirical equation which included an exponential decay (e.g., continuous absorption)

and a Gaussian (e.g., plasmon peak) functions, e.g., $A=a_{0}+a_{1} e^{-a_{2} \lambda}+a_{3} e^{-\frac{\left(\lambda-a_{4}\right)^{2}}{a_{5}}}$, where $A$ is absorbance, $\lambda$ is wavelength, and $a_{0}-a_{5}$ are fitting parameters.

Samples for TEM were obtained by evaporating a drop of the ageing solution on a TEM grid.

TGA analysis was performed using $10 \mathrm{mg}$ samples prepared by evaporating an appropriate amount of the ageing solution. The samples were heated at a rate of $10^{\circ} \mathrm{C} / \mathrm{min}$. 


\section{Kinetic study}

The ageing solution was prepared by dissolving butanethiol-protected $\mathrm{Au}$ nanoparticles in neat chlorobenzene to obtain $0.1 \mathrm{mM}$ solution. This solution was left to age at room temperature. The exchange reaction was initiated by mixing $0.1 \mathrm{~mL}$ aliquots of the ageing solution with $0.1 \mathrm{~mL}$ of $0.05 \mathrm{mM}$ solution of diradical disulfide ligand. The mixture was transferred to an EPR tube and reaction was monitored by EPR.

To test the effect of oxygen, a control reaction was performed in inert atmosphere. The reaction mixture was placed in an EPR tube, degassed/back-filled with Ar using three freeze-pump-thaw cycles, and kept under positive Ar pressure throughout the experiment. The absence of oxygen in the reaction mixtures was confirmed throughout the reaction by the narrow width of the EPR peaks (as the presence of paramagnetic oxygen broadens EPR signals).

\section{Kinetic model.}

Kinetic curves showed good fit to a simple exponential decay equation. Full description of the kinetic model is given below.

Reaction scheme (see also Ionita P., Caragheorgheopol A., Gilbert B.C., Chechik V. J. Am. Chem. Soc. 2002, 124, 9048):

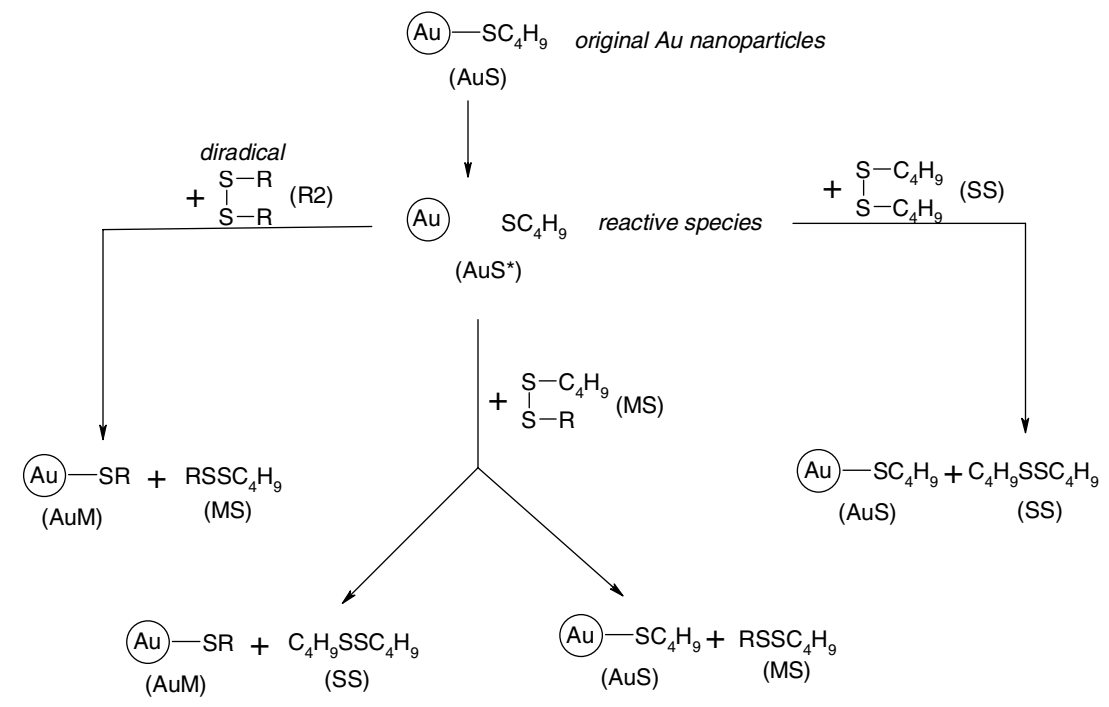

Kinetic equations (the abbreviations used in the equations are shown in the brackets in the scheme above): 


$$
\begin{aligned}
& \text { AuS } \stackrel{\mathrm{k}_{1}}{\longrightarrow} \text { AuS }^{*} \text { (rate-determining step) } \\
& \mathrm{AuS}^{*}+\mathrm{R} 2 \stackrel{\mathrm{k}_{2}}{\longrightarrow} \text { AuM }+\mathrm{MS} \\
& \mathrm{AuS}^{*}+\mathrm{MS} \stackrel{\mathrm{k}_{2} / 2}{\longrightarrow} \text { AuM }+\mathrm{SS} \\
& \mathrm{AuS}^{*}+\mathrm{MS} \stackrel{\mathrm{k}_{2} / 2}{\longrightarrow} \text { AuS + MS } \\
& \mathrm{AuS}^{*}+\mathrm{SS} \stackrel{\mathrm{k}_{2}}{\longrightarrow} \text { AuS + SS }
\end{aligned}
$$

Using steady-state approximation (if the first step is rate-determining), $\mathrm{d}[\mathrm{AuS} *] / \mathrm{dt}=0$

Applying this to the reaction scheme above,

$\mathrm{d}[\mathrm{AuS} *] / \mathrm{dt}=\mathrm{k}_{1}[\mathrm{AuS}]-\mathrm{k}_{2}[\mathrm{AuS} *][\mathrm{R} 2]-\mathrm{k}_{2}[\mathrm{AuS} *][\mathrm{MS}]-\mathrm{k}_{2}[\mathrm{AuS} *][\mathrm{SS}]=0$

Mass balance:

$[\mathrm{R} 2]+[\mathrm{MS}]+[\mathrm{SS}]=[\mathrm{R} 2]_{0}$, where $[\mathrm{R} 2]_{0}$ is initial concentration of the diradical.

Combining (1) and (2) gives $\mathrm{k}_{1}[\mathrm{AuS}]=\mathrm{k}_{2}[\mathrm{AuS} *][\mathrm{R} 2]_{0}$ or

$$
[\mathrm{AuS} *]=\frac{k_{1}[\mathrm{AuS}]}{k_{2}[\mathrm{R} 2]_{0}}
$$

Kinetic equation for the diradical is $\frac{d[\mathrm{R} 2]}{d t}=-k_{2} \times[\mathrm{AuS} *] \times[R 2]$. Combining this with (3) gives

$$
\frac{d[\mathrm{R} 2]}{d t}=-k_{1} \times[\mathrm{AuS}] \times \frac{[\mathrm{R} 2]}{[\mathrm{R} 2]_{0}}
$$

The kinetic equation (4) describes the concentration profile of diradical disulfide in the exchange reaction. One can see that integration of this kinetic equation assuming excess of $[\mathrm{AuS}]$ and constant $[\mathrm{R} 2]_{0}$ gives an exponential decay function. This justifies the use of exponential function to fit kinetic data.

On the other hand, the initial rate does not depend on the concentration of the diradical (as $[\mathrm{R} 2]=[\mathrm{R} 2]_{0}$ at $t=0$ and hence $\frac{d[\mathrm{R} 2]}{d t}=-k_{1}[\mathrm{AuS}]$ ). The reaction is therefore zeroth order with respect to the initial concentration of disulfide ligand.

Similarly, half-reaction time could be derived from the integration of equation (4) as $t_{1 / 2}=\frac{\ln 2 \times[\mathrm{R} 2]_{0}}{k_{1}[\mathrm{AuS}]}$. One can see that half-reaction time is linearly proportional to the initial concentration of disulfide $[\mathrm{R} 2]_{0}$, typical of zeroth order reactions.

\section{References.}

[1] T. G. Schaaff, M. N. Shafigullin, J. T. Khouri, I. Vezmar, R. L. Whetten, W. G. Cullen, P. N. First, C. Guttiérrez-Wing, J. Ascensio, M. J. Jose-Yacamán, J. Phys. Chem. B., 1997, 101, 7885. 
[2] M. J. Hostetler, J. Wingate, C.-J. Zhong, J. E. Harris, R. W. Vachet, M. R. Clark, J. D. Londono, S. J. Green, J. J. Stokes, G. D. Wignall, G. L. Glish, M. D. Porter, N. D. Evans, R. W. Murray, Langmuir, 1998, 14, 17. 